\title{
A Microfluidic Gradient Generator to Simulate the Oxygen Microenvironment in Cancer Cell Culture
}

Louise Orcheston-Findlaya ${ }^{*}$, Azadeh Hashemia ${ }^{a}$ Ashley Garrill ${ }^{b}$, Volker Nock ${ }^{a}$

*louise.orcheston-findlay@pg.canterbury.ac.nz

aThe MacDiarmid Institute for Advanced Materials and Nanotechnology, Department of Electrical and Computer Engineering, University of Canterbury, NZ

${ }^{b}$ The School of Biological Sciences, University of Canterbury, NZ

Department of Electrical and Computer Engineering, University of Canterbury, Private Bag 4800, Christchurch, 8140, New Zealand

School of Biological Sciences, University of Canterbury, Private Bag 4800, Christchurch, 8041, New Zealand

\section{Abstract}

Standard cancer lab models lack many attributes of the in-vivo cancer microenvironment. Oxygen levels for example are not commonly controlled in 2D cell-culture well plate experiments. However, low $\mathrm{O}_{2}$ (hypoxia) in particular is common in cancerous tissue due to high proliferation rates of cancer cells and inadequate vasculature. Hypoxia is also associated with cancer recurrence and drug resistance. We report a microfluidic system capable of exposing a $2 \mathrm{D}$ cell-culture to a dissolved oxygen gradient ranging from hypoxia $(<1 \mathrm{mg} / \mathrm{L})$ to hyperoxia $(40 \mathrm{mg} / \mathrm{L})$ for over 30 minutes, measurable in real-time using an integrated sensor film. The film incorporates a photostable, non-cytotoxic oxygen-sensitive fluorescent dye, which exhibits a linear response and high contrast ( $I_{0}$ $/ /_{100}=12$ ) within the range of interest, was integrated onto glass substrates as a cell culture substrate. To demonstrate the applicability of the platform, Ishikawa cancer cells were cultured on the platform and exposed to linear cross-stream oxygen gradients. The platform provides a valuable tool for the culture of cancer cells in an in-vivo like microenvironment and will enable more accurate screening of new anti-cancer drugs.

\section{Keywords}

Microfluidics, oxygen, cancer, fluorescent microscopy, gradient generator, thin film sensor 


\section{Introduction}

The Importance of Oxygen Conventional culture models, such as two-dimensional (2D) cell mono-layers in culture plates or wells, lack many environmental features that are present in-vivo. This is concerning given the inhomogeneous oxygen microenvironment present in cancer tumours.

Hypoxia is often present in-vivo due to high proliferation rates and inadequate or absent vasculature [1]. Hypoxic conditions have been correlated with the development of aggressive phenotypes, cancer recurrence [2], and shorter life span before recurrence [3].

Hypoxia is difficult to define as the oxygen tension in various tissues throughout the body varies. Table 1 shows the concentration of oxygen of several types of healthy tissue and their cancerous counterparts [4], indicating that disease can have as little as $5 \%$ the oxygen of healthy tissue. Hypoxia in endometrial cells is considered $1-1.5 \mathrm{mg} / \mathrm{L}$ [5].

The absence of oxygen control in anti-cancer drug discovery and screening systems is an issue that needs to be urgently addressed. The lab model needs to be upgraded to include at least some of the nuances of the 3D environment. Naturally, this has led to the use of 3D cell culture, and 2D culture with environment control on the macro- or microscale.

Table 1: Oxygen concentration present in healthy tissue and their diseased counterparts. Adapted from $[4,5]$.

\begin{tabular}{|l|l|l|}
\hline \multicolumn{1}{|c|}{ Tumour type } & \multicolumn{1}{|c|}{$\begin{array}{c}\text { Median } \mathrm{O}_{2}(\mathrm{mg} / \mathrm{L}) \\
\text { healthy }\end{array}$} & \multicolumn{1}{c|}{$\begin{array}{c}\text { Median } \mathrm{O}_{2}(\mathrm{mg} / \mathrm{L}) \\
\text { diseased }\end{array}$} \\
\hline Head and neck & 6.9 & 2.0 \\
\hline Lung & 5.1 & 1.0 \\
\hline Pancreatic & 6.9 & 0.4 \\
\hline Cervical & 6.9 & 0.4 \\
\hline Prostate & 7.0 & 2.7 \\
\hline endometrial & $19-20$ & $1-1.5$ \\
\hline
\end{tabular}

$3 D$ cell-culture The use of microscale $3 D$ cell clusters $[6,7]$ and $3 D$ hydrogels encapsulated cells [8] has been on the rise due to the ability of these technologies to better reproduce the 3D microenvironment and thus provide more indicative results in relation to drug efficacy [9]. While these methods are 3D, the oxygen inhomogeneity is uncontrolled, size reproducibility can be poor [10], and it is difficult to visualise the inner hypoxic areas until after the microtumour/3D scaffold has been fixed and sectioned, or the cells lysed.

There are many advantages to environmental control on-chip, such as fast and relatively cheap prototyping for lab-on-a-chip devices, rapid and controlled cycling of temperature, media and concentration gradients. Unique to oncology is the opportunity to investigate the use of rare primary cells towards future personalised drug screening processes. 
Specifically for the 2D culture on-chip, it is possible to observe cell-cell interactions, migration and other biological processes in a microchannel towards a real tumour-on-achip model.

$2 D$ cell-culture on-chip To date, a limited number of studies have used oxygen control and sensing alongside 2D cancer cell-culture and drug testing on the microscale $[11,12]$. Two-dimensional systems allow for real-time imaging of cells, while microfluidics can be used to simultaneously mimic aspects of the 3D microenvironment and to guide cell-cell interactions. The added advantage over 3D cultures is the level of control and the ability to incorporate or exclude attributes of the 3D environment as desired.

Oxygen scavenging/generating reactions for oxygen control [11] or gas channels separated by a thin membrane, either in 2D $[12,13]$ or 3D [14] have been commonly used to control $\mathrm{O}_{2}$. For oxygen measurement, oxygen sensitive dyes [12], or fluorescent films $[15,16]$ can be used. The dyes are dissolved in the cell-culture media, with effects of dye uptake on drug response, cell viability, proliferation and metabolism mostly unknown. The films are fabricated by typically encapsulating a sensing compound in a solid-state matrix, and do not come into contact with the cells or media.

Drug screening and discovery Lab model systems that mimic the 3D environment, when used in drug screening, could reduce the occurrence of false positives and negatives. In addition to that, the visibility of cells allows quick assessment of drugs without the complex lysing, or sectioning and staining process necessary for 3D cultures. Additionally, with the utilisation of high throughput parallelisation possible with microfluidic devices, the process could be sped up considerably.

$2 D$ culture on chip with oxygen control In this work, we present a reusable, reversiblysealed microfluidic system capable of producing an oxygen gradient, as illustrated in Fig. 1. Non-invasive oxygen sensing functionality was embedded into the cell-culture substrate and off-chip flow and gas control was employed. The oxygen sensor, previously developed in our group $[15,16]$ consist of a polystyrene (PS)-based matrix incorporating platinum(II) octaethylporphyrin ketone (PtOEPK) as an oxygen-sensitive fluorescent dye. This allows intensity-based fluorescent imaging as a function of oxygen concentration gradients through adherent cells in real time [17]. Regarding the microfluidic chip, a reusable, resealable approach was used to allow further biological analysis. A plug and play approach was used to provide gas control for ease of prototyping and system modification. We demonstrate applicability of the platform by culturing Ishikawa endometrial cancer cells and exposing the culture to cross-stream oxygen gradients onchip. 


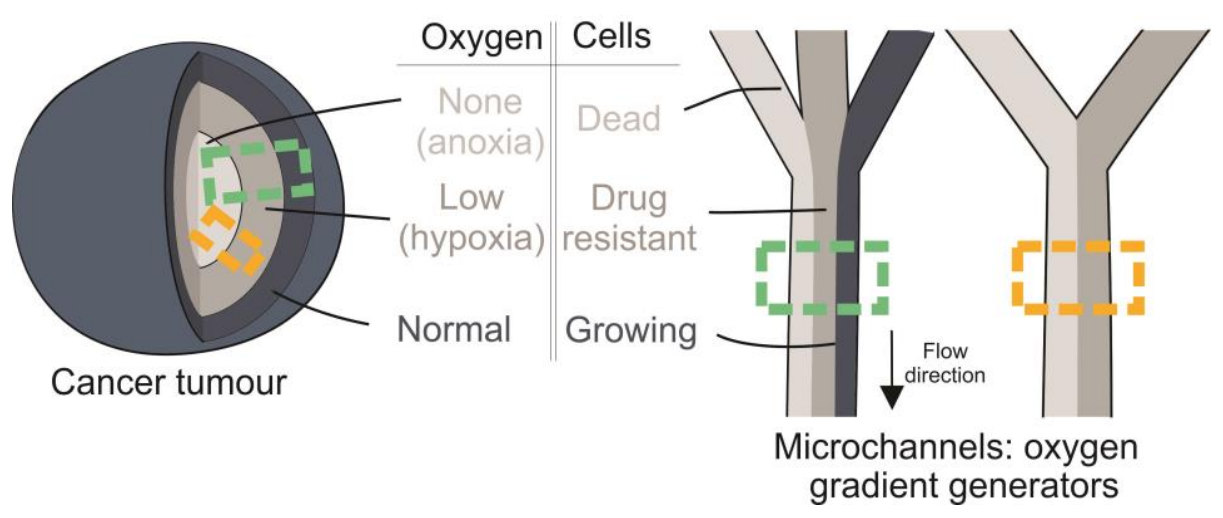

Figure 1: A schematic representation of the inhomogeneous oxygen concentration gradients present in the cancer microenvironment alongside the equivalent microfluidic oxygen gradient generators.

The reported system enables the generation of a gradient between the extremes of hyperoxia and hypoxia. The gas control system, at present, ensures near-maximum saturation of the cell-culture media with oxygen/nitrogen for hyperoxia and hypoxia, respectively.

\section{Materials and Methods}

The geometry of the microfluidic chip used to generate an oxygen gradient is shown in Fig. 2a). Two inlet channels converged on a $1 \mathrm{~mm}$ wide, $100 \mu \mathrm{m}$ high, $3 \mathrm{~cm}$ long cellculture channel, which then diverges to three $333 \mu \mathrm{m}$ wide outlet channels. Standard photolithography and replica moulding techniques were used to form a mould and subsequent microfluidic chips. Briefly, a dehydrated silicon wafer was laminated (Sky 335R6, DSB) with a $100 \mu \mathrm{m}$ negative-tone dry film of photoresist (SUEX, Microlaminates) at $60^{\circ} \mathrm{C}$. The channel geometry was designed using L-Edit (Mentor Graphics) and a chrome-covered glass plate was exposed ( $\mu \mathrm{PG}$, Heidelberg), developed and etched to reveal this pattern. The photoresist was then exposed to $675 \mathrm{~mJ} / \mathrm{cm}^{2}$ of ultraviolet (UV) light (MA-6, Suss Microtec) through the mask patterns with 20 rounds of $10 \mathrm{~s}$ exposure and $60 \mathrm{~s}$ of no exposure between rounds. The wafer was subsequently heated at $60^{\circ} \mathrm{C}\left(100^{\circ} / \mathrm{h}\right.$ ramp $)$ for $5 \mathrm{~min}, 90^{\circ} \mathrm{C}\left(100^{\circ} \mathrm{C} / \mathrm{h} \mathrm{ramp}\right)$ for $10 \mathrm{~min}$, then cooled to room temperature $\left(15^{\circ} \mathrm{C} / \mathrm{h}\right.$ ramp). Mould development was then performed by immersion in Propylene glycol monomethyl ether acetate (PGMEA) for $10 \mathrm{~min}$ and rinsed with isopropyl alcohol. 
a)

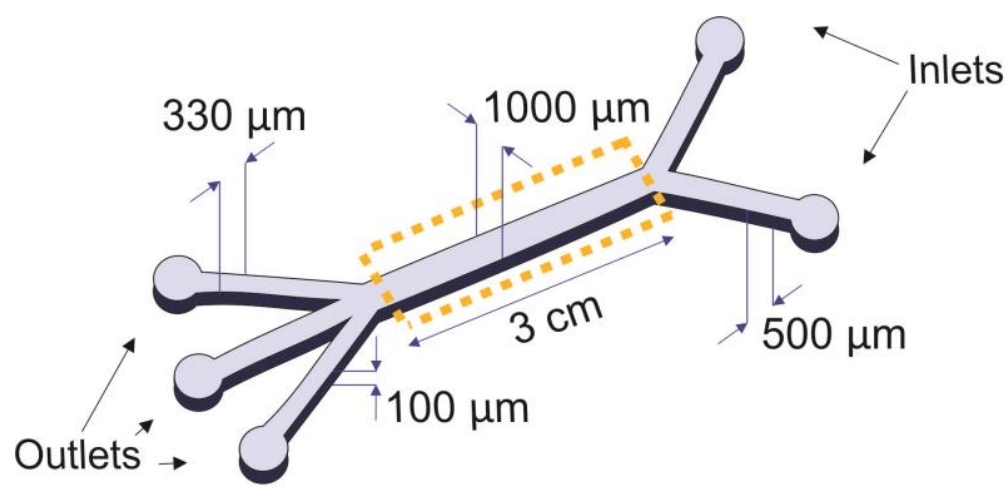

b) Viewing window

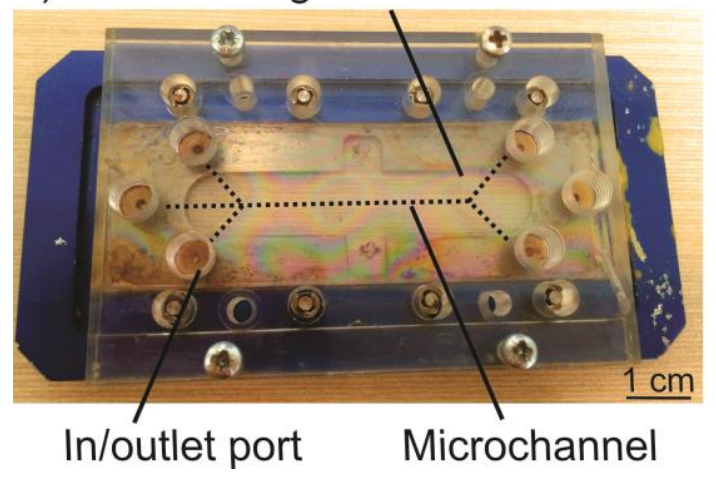

c)

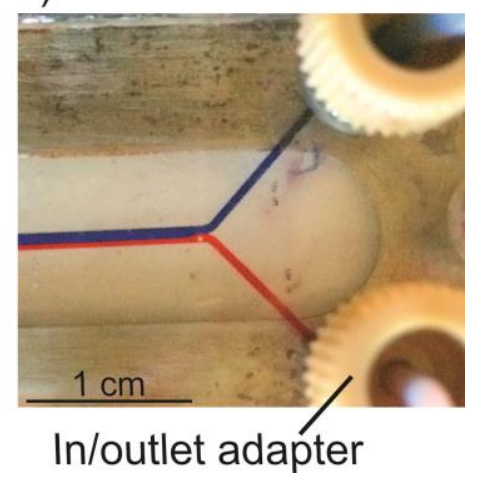

Figure 2: A schematic of the channel geometry, and a photograph of the chip holder, and the channels filled with food dye.

Polydimethylsiloxane (PDMS) prepolymer was mixed with curing agent $(10: 1 \mathrm{w} / \mathrm{w}$, Sylgard 184, Dow Corning) and desiccated before pouring into a $5 \mathrm{~mL}$ syringe and further desiccated. The degassed PDMS was then injected onto the precut Si/SU-8 mould set into a casting station (Microfluidic ChipShop), and cured at $80^{\circ} \mathrm{C}$ for $4 \mathrm{~h}$. The casting station top plate with PDMS was then peeled off the mold, and transferred to the chip holder, shown in Fig. 2b).

The sensor film substrate was prepared by dissolving PS pellets and PtOEPK powder unto toluene. $7 \% \mathrm{w} / \mathrm{w}$ of PS/toluene was first prepared and left for the PS to dissolve for $>24 \mathrm{~h}$. Powdered PtOEPK (company, catalogue number) was then added $(1 \mathrm{mg} / \mathrm{ml}$ ) and placed in an ultrasonic bath for 15 minutes at room temperature.

The sensor films were spin-coated onto glass microscope slides that had been dehydrated for $>24 \mathrm{~h}$ at $180^{\circ} \mathrm{C}$ and cleaned in $100 \%$ oxygen plasma (Emitech, K1050X) for 10 minutes at $100 \mathrm{~W}$. Approximately $200 \mu \mathrm{L}$ of sensor solution was pipetted onto each microscope slide in the first stage of a two-stage spin program: (i) $4000 \mathrm{rpm}$ for $5 \mathrm{~s}$ (1000 rpm ramp) and (ii) $8000 \mathrm{rpm}$ for $55 \mathrm{~s}$ (500 rpm ramp). After spin coating, the films were stored in the dark while the toluene was allowed to evaporate $(>24 \mathrm{~h})$ then annealed for $90 \mathrm{~s}$ at $100^{\circ} \mathrm{C}$. 
Each inlet stream, oxygenated (hyperoxic) and nitrogenated (hypoxia), had its own line of components, as shown in Fig. 3. Each line consisted of rigid, low gas-permeability polytetrafluoroethylene (PTFE) tubing (ID 1/16"), flanges and connectors, flow restrictor, bubble trap, flow sensor (FS1, Elveflow) and homemade gas exchanger. A pressuredriven flow controller (OB1, Elveflow) provided fluid flow. An oxygen measurement module (PreSens) with one flow-through sensor for each inlet stream was used for sensor film calibration.

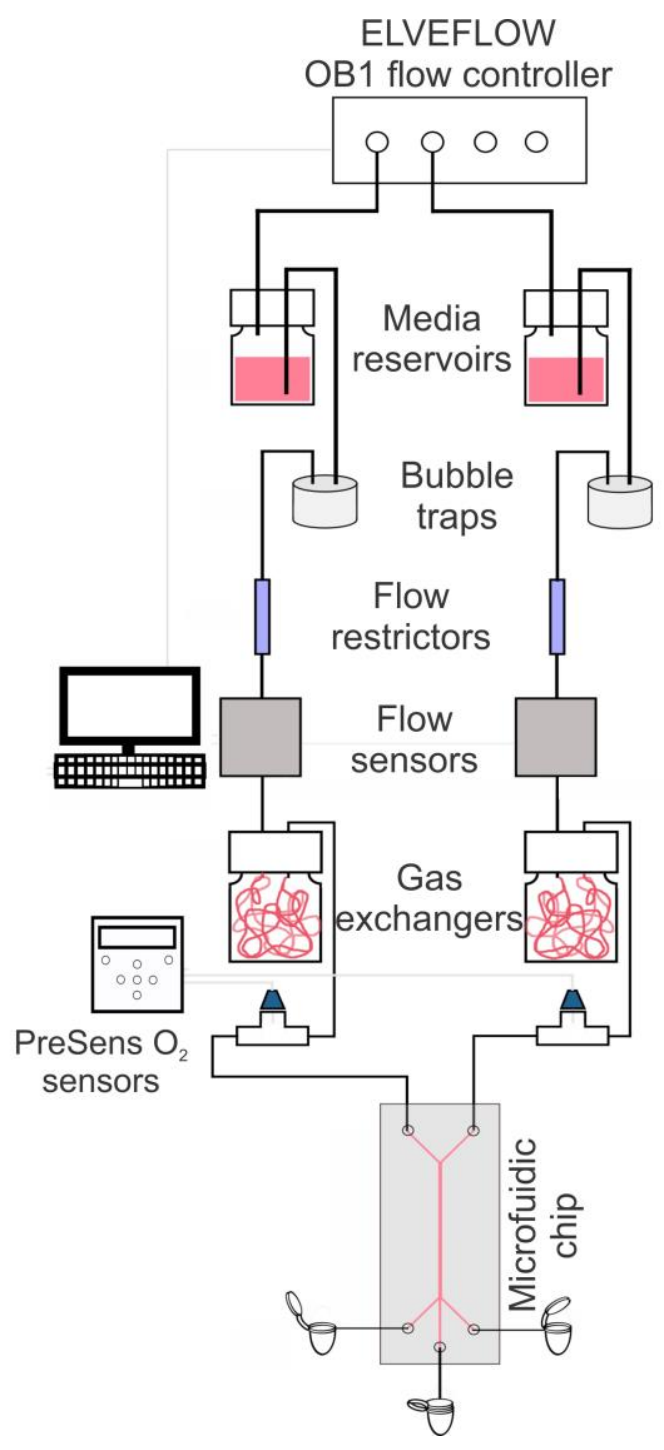

Figure 3: A schematic representation of the oxygen control and measurement system used to generate the oxygen gradients on chip.

Dissolved oxygen (DO) in the fluid was controlled with gas exchangers fabricated from plastic bottles. Low gas permeability tubing outside the bottle was interfaced with high gas permeability tubing inside (Silastic, Dow Corning, length approx. $3 \mathrm{~m}$ ) through the bottle lid. Each gas exchanger was pressurised with either oxygen or nitrogen (technical 
grade, Southern Gas) to provide hyperoxia and hypoxia, respectively. Gas flow exiting the gas exchangers was limited by flowmeters (VFB-60-SSV, Series VF, Dwyer).

To facilitate cell attachment, sensor films were coated with poly-L-lysine (Sigma Aldrich). A central rectangle of approximately $3 \mathrm{~cm}^{2}$ was defined on each film with a wax pencil. Stock $10 \mathrm{x}$ poly-L-lysine solution was diluted to $1 \mathrm{x}$ with phosphate-buffered saline (PBS) (Sigma Aldrich) and dispensed onto this. After $5 \mathrm{~min}$, films were rinsed with fresh PBS, incubated for $>2 \mathrm{~h}$ at $37^{\circ} \mathrm{C}$ and either used immediately, or stored at $4^{\circ} \mathrm{C}$.

Ishikawa endometrial cancer cells [18] were cultured, trypsinised, pelleted and resuspended in working media consisting of Minimum Essential Media (MEM, Thermofisher) supplemented with $10 \%$ Fetal bovine serum (FBS, Thermofisher), $1 \%$ Penacilin-Streptomycin (Thermofisher), and $0.4 \%$ Fungizone (Sigma Aldrich). A suspension of $10^{4}$ cells was dispensed on each slide and incubated at $37^{\circ} \mathrm{C}$ for $>48 \mathrm{~h}$. Media was changed every $>12 \mathrm{~h}$. This resulted in a $>60 \%$ confluent cell monolayer.

The flow system was assembled without the substrate and pre-sterilised with $70 \%$ ethanol by flow-through. It was then sterilised further under UV light overnight, with chip holder components and tools alongside. Each tubing line and media reservoir was filled with working media using a syringe and transferred to a microscope (Eclipse 80i, Nikon). The media reservoirs were connected to the flow controller, and the outlets were directed towards a waste container. The nitrogen and oxygen regulators were pressurised to $20 \mathrm{kPa}$ and $5 \mathrm{kPa}$, respectively, and the flow meters set to $150 \mathrm{cc} / \mathrm{min}$. Software (SDK, Elveflow) was set to maintain a flow rate of $80 \mu \mathrm{L} / \mathrm{min}$, while gas saturation was in process and the oxygen gradient was being established. The oxygen measurement apparatus was used to record the DO concentration in $\mathrm{mg} / \mathrm{L}$.

Once the DO gradient was defined, the substrate and cells were placed in the chip holder base. The PDMS chip was then brought into contact and screwed tight, leaving only the cells in the channel intact. The chip was transferred to a heated microscope stage set to $37^{\circ} \mathrm{C}$, flow was reduced to $5 \mu \mathrm{L} / \mathrm{min}$ and both tubing inlets were attached to the inlet chip holder ports.

A camera (ORCA-Flash4.0 V2, Hamamatsu) and custom filter cube (EX595/ EM760, Chroma) were used for image acquisition with the HClmage Live (Hamamatsu) software suite. Automated image sequencing was used to capture images every second for the duration of gradient exposure - typically between 1 and $2 \mathrm{~h}$. These images were used to produce videos and cross-sections of the fluorescent signal using the image processing package in MATLAB (2017a, Mathworks).

A MATLAB script was written to first extract each image in each sequence of images, typically at 1 frame per second, producing hundreds of images. This sample rate was maintained in order to capture any rapid or short-lived changes in the oxygen gradient. The MATLAB script began by defining a region of interest $(\mathrm{ROI})$ that included everything inside the channel walls and set all other pixels to zero. This ensured that the graphs generated only applied to the 
channel to the exclusion of all other intensity data. Typically 6 locations were selected along the length of the visible section of microchannel. To probe the cross-stream intensity gradient at these locations, the intensity values of each pixel along each of the 6 cross-sections were extracted for each frame in the series and stored. Because the cross-stream intensity gradient was expected to be effected by photobleaching, the mean, minimum and maximum cross-stream gradient were calculated for visualisation. The frame in which the maximum and minimum gradient were captured was determined by a summation of the pixels in each cross-section and the extraction of the maximum and minimum.

This method of gradient extraction from the imaged film was such that every pixel was measured and graphed. This, in our case, has some disadvantages because of the sometimes inhomogeneous brightness of the film over short distances $(10 \mu \mathrm{m})$ due to partially dissolved PtOEPK particles. As the main function of sensor film was to resolve the shape of the oxygen gradient, however, this does not effect the usability of the film for resolving the gradient shape.

The intensity extracted from each image was converted to DO concentration in $\mathrm{mg} / \mathrm{L}$ using a simple conversion. The minimum and maximum intensities given on the crosssection closest to the start of the cell-culture channel were set to the incoming $\mathrm{O} 2$ values given by the flow-through sensors. This allowed for the most accurate conversion least effected by cross-stream diffusion and compared well to calibration of each film before use

\section{Results and Discussion}

Microfluidic devices promise to provide a means to bridge the micro-environmental gap between simple static well culture and complex 3D culture systems. Laminar flow-based culture chambers allow one to establish gradients in nutrient and gas supply to 2D cell sheets, thus mimicking gradients observed in 3D cellular constructs. The ability to expose 2D cells to more complex micro-environments will make results obtained with these more comparable to in-vivo, while retaining many of the advantages of this traditional culture setup. The successful culture of cancer cells in microfluidic chips requires a fine balance of nutrients, gas supply and flow parameters. As opposed to static culture well plates, cells in microfluidic channels are exposed to fluid flow and thus shear-stress. While the latter limits the rate at which fluid can be supplied to cells before they detach, it is the presence of laminar flow inside the devices that allows one to establish and control concentration gradients.

To test our platform, we first characterized the dynamics and range of DO generation in the microfluidic chip using the external gas exchangers. An example of the DO concentration and the pressure in each gas exchanger during gradient setup is shown in Fig. 4. With an applied flow speed of $50 \mu \mathrm{L} / \mathrm{min}$, stable hypoxia as found in endometrial cancer tissue $(<1 \mathrm{mg} / \mathrm{L})$ was produced with the nitrogenated gas exchanger after 
approximately $3 \mathrm{~h}$, and hyperoxia at $40 \mathrm{mg} / \mathrm{L}$ after around $1.5 \mathrm{~h}$ with the oxygenated gas exchanger. This suggests that oxygen dissolves more readily than nitrogen at the same pressure in working media. This is to be expected, as the solubility of oxygen in water is approximately twice that of nitrogen [19]. The delay between a change in the gas exchanger pressure and the associated concentration change was due to the slow flow rate and the distance of the flow-through sensors from the gas exchanger. We found that the flow rate had to be kept at or below $10 \mu \mathrm{L} / \mathrm{min}$ to prevent excessive shear-stress from detaching Ishikawa cells.

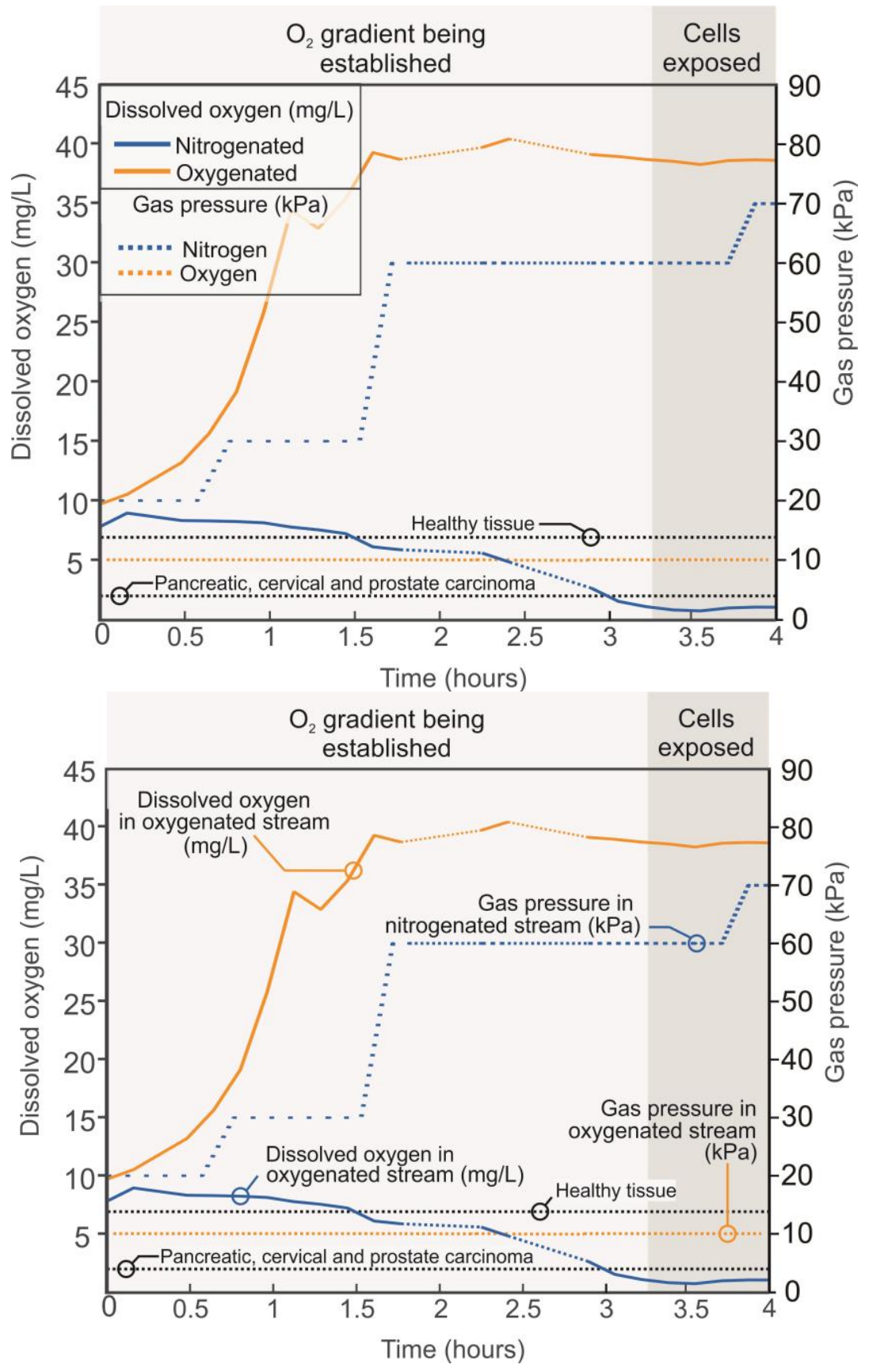


Figure 4: The relationship between the gas pressure (right axis, $\mathrm{kPa}$ ) and oxygen concentration (left axis, mg/L) over time. Orange lines represent the pressure/DO (dashed/solid lines) of the oxygenated stream and blue lines represent the pressure/DO (dashed/solid lines) of the nitrogenated stream. Black, horizontal dashed lines denote the oxygen concentration in typical healthy and diseased endometrial, cervical, prostate and pancreatic tissue. Dashed lines in the dissolved oxygen data represent intermissions in the data collected.

To further establish the dynamics of DO generation, we compared the results obtained with cell-culture media with water. The salinity of the cell culture media was $10 \mathrm{mg} / \mathrm{kg}$ [21]. Incorporating pressure, salinity and temperature, the DO concentration in a water solution with the same salinity and oxygen saturation at sea level is predicted to be $8.57 \mathrm{mg} / \mathrm{L}$ at $20^{\circ} \mathrm{C}$, and $6.38 \mathrm{mg} / \mathrm{L}$ at $37^{\circ} \mathrm{C}$, respectively [20]. At the start of data acquisition, the measured DO concentration was slightly higher at $9.3 \pm 0.7 \mathrm{mg} / \mathrm{L}(\mathrm{n}=5)$. As the media traversed the chip, it was heated to $37^{\circ} \mathrm{C}$ by the stage heater. This temperature rise decreases the solubility of the media and thus the predicted DO concentration reduced to $6.38 \mathrm{mg} / \mathrm{L}$. This may mean that the DO present in the hypoxic stream was lower than the $1 \mathrm{mg} / \mathrm{L}$ measured at room temperature, even when considering the slightly higher measured value in atmospheric conditions, compared to what was predicted. If required, this can be further quantified by calibrating the PS/PtOEPK sensor film, prior to cell imaging, with hyperoxic and hypoxic working media at $37^{\circ} \mathrm{C}$ deposited in wells separate from the channels immediately before attaching each inlet stream tubing.

To simplify the setup, the concentration of dissolved $\mathrm{CO}_{2}$ was not controlled in this work. It is typically supplied at $5-10 \% \mathrm{CO}_{2}$ to maintain $\mathrm{pH} 7.4$ in traditional incubation systems. While the absence of $\mathrm{CO}_{2}$ could affect the viability and behaviour of the cells, emphasis of our work at this stage is to establish differences between cells cultured on the platform under hypoxic and hyperoxic conditions. In the future, and to compare our results to static well-based culture, we aim to address this by either modifying the gas control system to include $\mathrm{CO}_{2}$, or by use of cell-culture media with added Earle's salts, which does not require the addition of gaseous $\mathrm{CO}_{2}[21]$.

Hypersonic and hyperoxic flow streams were combined inside the microchannel and the resulting gradients were characterized using the integrated oxygen sensor film. Figure 5 shows an example of an established cross-stream oxygen gradient inside the main culture channel. Due to the slow flow rate used, the cross-stream oxygen concentration decreases approximately linearly from the hyperoxia stream side to the hypoxia stream side. The slope of the profiles gradually decreases from the inlet to the outlet end of the main channel, as characteristic for diffusion controlled mixing. This is slightly complicated by the fact that, the further from the start of the cell channel, the more oxygen the PDMS itself is able to give/receive through the channel walls. Along with cross-stream diffusion, this results in a smoother DO gradient. This linear gradient, however, is advantageous as it arguably produces an environment more similar to the in-vivo oxygen environment than step-wise gradients typical at higher flow rates.

The use of this system for cell culture was validated with the growth of Ishikawa human endometrial cancer cell line. These cells represent a well differentiated endometrial adenocarcinoma cell line with the special characteristic of bearing estrogen and 
progesterone receptors [18]. Ishikawa cells provide a good model system, as cells can be grown in 2D and 3D, with distinct differences in drug resistance depending on morphology [22]. A monolayer of these cells was exposed to the $\mathrm{O}_{2}$ gradient for 30 40 minutes. The hypoxic stream at its lowest saturation produced $<1 \mathrm{mg} / \mathrm{L}$. This is below both the $5 \%$ where hypoxia generally occurs in mammalian tissue [2], and below that of endometrial carcinoma, which typically contain $1-1.5 \mathrm{mg} / \mathrm{L}$ [5]. Furthermore, a region at the hypoxic edge of the channel approximately $250 \mu \mathrm{m}$ wide was under hypoxia $(<5 \mathrm{mg} / \mathrm{L})$, and the side-most $100 \mu \mathrm{m}$ of that was under conditions suitable for hypoxic studies with endometrial cancer $(<1.5 \mathrm{mg} / \mathrm{L})$. These regions are $0.8 \mathrm{~cm}$ from the start of the cell-culture channel.

The hyperoxic stream, at its highest, produced $40 \mathrm{mg} / \mathrm{L}$. Even though hyperoxia has no defined upper limit, this was not deemed physiologically relevant, but could be made so with small changes to the gas control system. The hypoxic region, however, is the more important in a system designed for hypoxic studies and the more difficult to produce experimentally in a conventional well plate system.

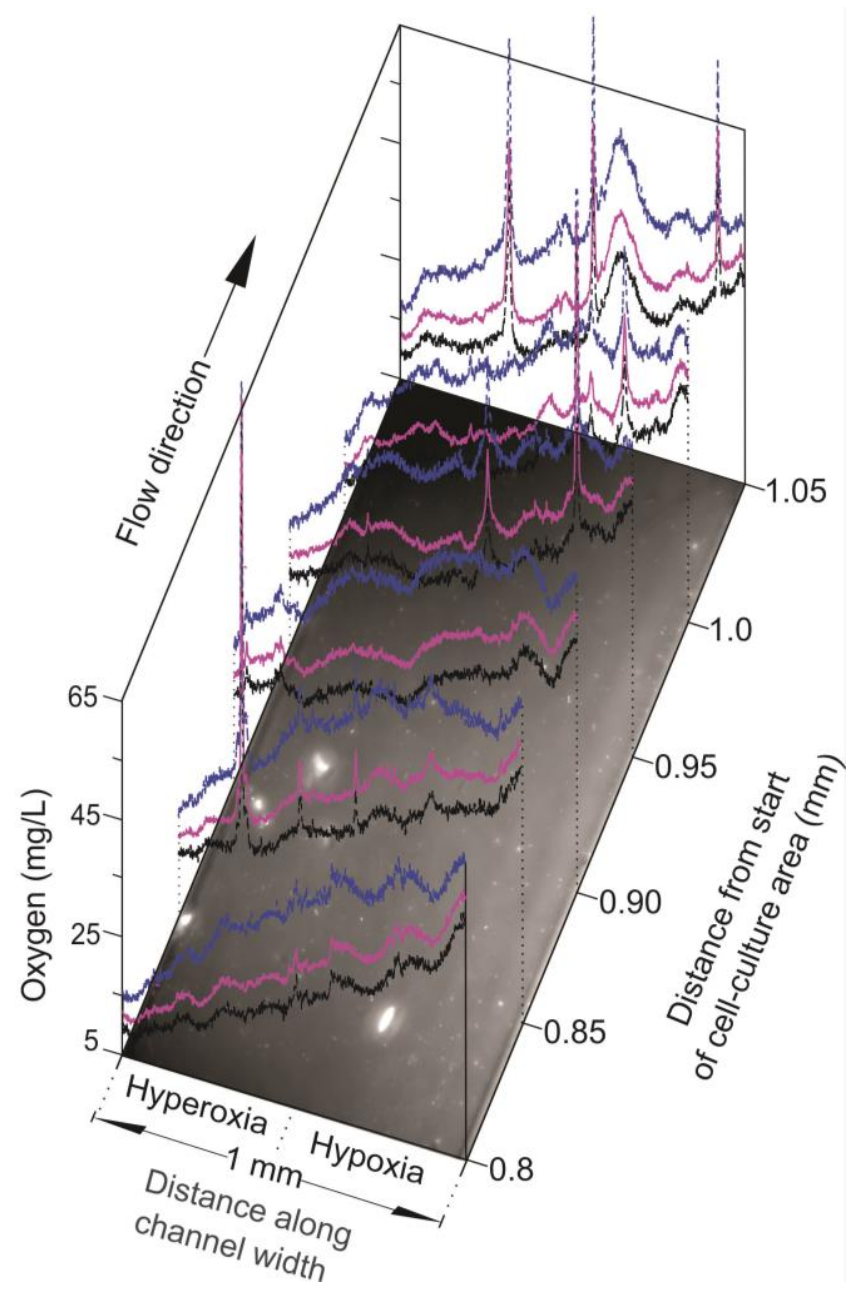

Figure 5: A 3D graph showing the mean (purple), maximum (blue) and minimum (black) crosssectional oxygen gradient at several positions along a section of the channel length over 30 
minutes. The intensity of the sensor film at the start of data acquisition is shown beneath the traces, white and black denoting low and high oxygen concentrations, respectively.

The mean, maximum and minimum DO gradients at several points in the channel over 30 minutes are shown in Fig. 5. The video version of this graph (see ESI) shows that the integrated sensor film is capable of visualising and tracking even the smallest instabilities in the gradient, caused by variations in the flow control, with high lateral resolution in realtime. Some photo-bleaching of the film can also be observed in this example in the form of the intensity gradient decreasing, but maintaining its shape over time. During experimentation, illumination is usually limited to data acquisition during fluorescent imaging to reduce this effect. A typical fluorescent image of the channel area is shown below the cross-sections. Other systems have produced a smoother oxygen gradient [11, 12], but the gradient profile is dependent on flow speed. Restrictions are imposed on the possible gradient profiles, because the shear exerted on cells must be kept low. It is possible to produce varied oxygen gradients with a constant low $5 \mu \mathrm{L} / \mathrm{min}$ flow speed with our system, and the stability can be improved with modifications to the flow control system. Other work, using sodium sulfite, has shown the production of a hypoxic laminar flow stream with $0.5 \mathrm{mg} / \mathrm{L}$ of oxygen [11]. This, however, was approximately $30 \%$ higher than that in the lung carcinoma cells used and approximately $70 \%$ higher than the cervical cancer cells used (Table 1). Another system has previously been used to firstly expose alveolar epithelial cells to an oxygen gradient between $9 \mathrm{mg} / \mathrm{L}$ and $21 \mathrm{mg} / \mathrm{L}$ oxygen, and secondly between $0.5 \mathrm{mg} / \mathrm{L}$ and $21 \%$ [12]. This system, however, used oxygen sensitive dye for measurement. While both these systems produced gradients that are more stable than our system at present, there are additional advantages such as the re-usability, gradient versatility and the ability to be upscaled that our system offers.

We observed previously that cells do not adhere readily to the native (used after annealing with no modifications) PS/PtOEPK sensor film, as it is hydrophobic [23]. When culturing cells in microchannels, usually a cell attachment protein such as collagen [17], fibronectin [11,12] or hydrogel [14] is used to facilitate cell attachment and spreading. However, the acetic acid solution used to dissolve collagen for example was found to lift off the thin PS/PtOEPK from the glass beneath. However, we found it is possible to encourage strong cell adherence first by treating the native films with plasma, then with a hydrophilic polymer polyvinylpyrrolidone [23]. For the current work we used a second alternative method, which involved treating the native films with poly-L-lysine. This was also found to be effective at providing a bioactive surface for the cells to adhere to.

The shear in the channel during continuous perfusion at $10 \mu \mathrm{L} / \mathrm{min}$ was less than $0.03 \mathrm{dyne} / \mathrm{cm}^{2}$. This is well within what is considered an acceptable range for adherent cells - 0.3 dyne $/ \mathrm{cm}^{2}$ is considered the maximum [24]. It was found that the shear had more of an effect on cells, even at a slow $5 \mu \mathrm{L} /$ min flow speed, when confluent. This was likely because there were some cells that were adhered partially to the sensor film and partially to adjacent cells, making the fluid more likely to wash cells away. This lead to the reduction in the original cell-seeding density, which caused the movement of cells to be virtually undetectable by the human eye (see ESI). 
A section of a monolayer of Ishikawa cells $1 \mathrm{~cm}$ from the start of the cell-culture channel is shown in Fig. 6. After 30 min of hypoxia/hyperoxia exposure, the cells in hyperoxia appear more rounded and raised - a sign of weakening attachment or apoptosis - than the cells under hypoxia. This is unsurprising as hyperoxia of this degree would produce free radicals that cause DNA damage to cells likely to cause cell death. This after all is the mechanism that many anti-cancer drugs used to treat cancer, and is believed to be one of the reasons that hypoxia causes drug resistance [25].

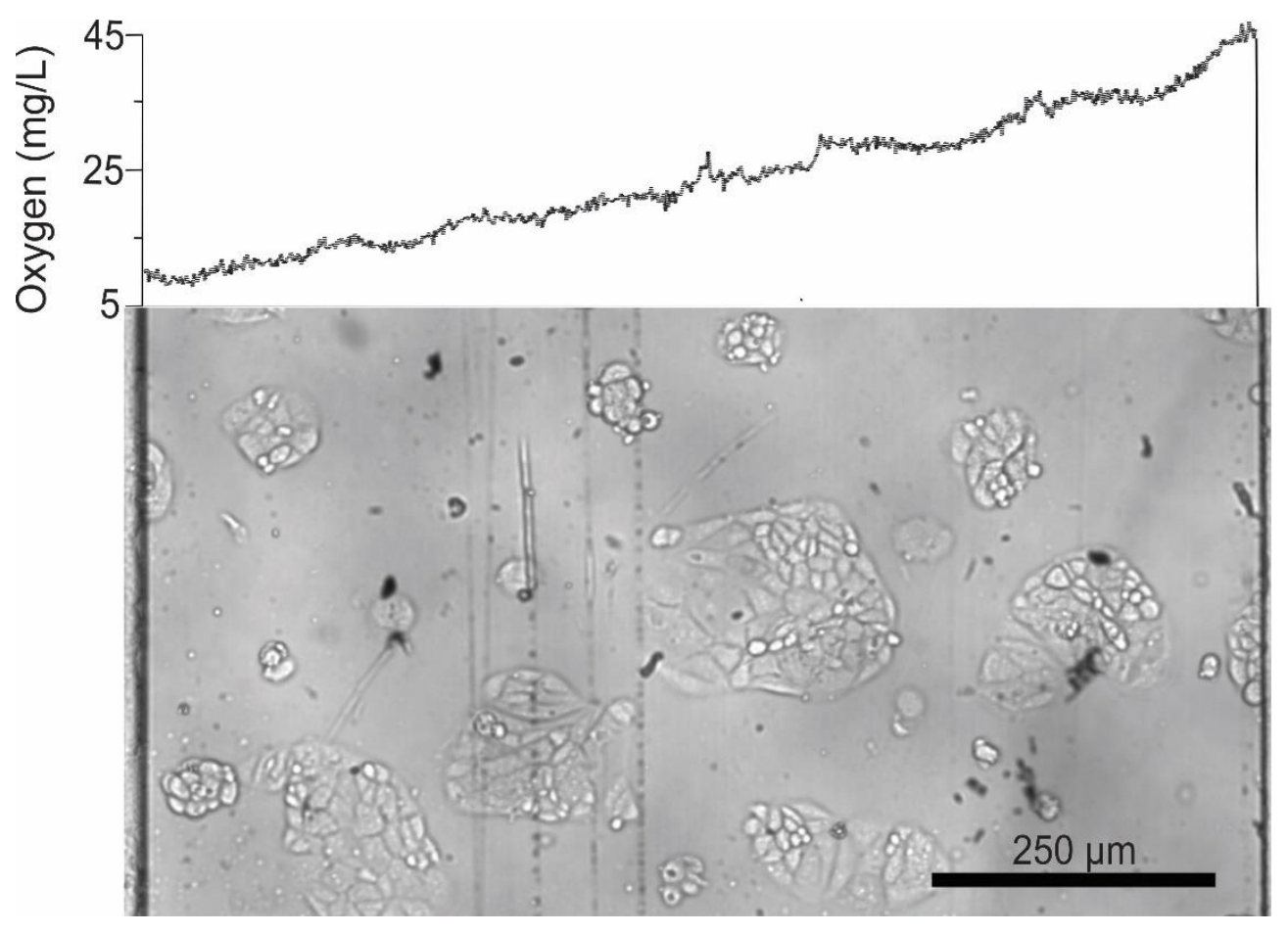

Figure 6: The upper trace shows an example of the intensity gradient extracted from one second in the 40 minutes that these cells were exposed, and converted into the DO concentration. The lower image shows a light microscope image of several 2D collections of Ishikawa endometrial cancer cells adhered to the PS/PtOEPK sensor film showing their morphology after 40 minutes in the oxygen gradient.

There were no visible morphology changes in the hypoxic region. There is a possibility, however, that there are nanoscale changes activated by hypoxia such as the formation of pores in the cell membrane brought about by the increase in vascular endothelial growth factor [26]. Investigation of nanoscale properties is possible with our system because it is reversibly sealed. The PDMS can be lifted off, fixing the cells and imaging the cells at the nanoscale with atomic force microscopy (AFM). Furthermore, we are currently in the process of combining cell-culture under oxygen gradient on our platform with live staining for hypoxia-inducible factors to visualise changes in cell metabolism due to the micro-environment the cells are exposed to. In the future, anti-cancer drugs will be included into the flow streams to study the role of oxygen concentration on resistance to treatment and compare this to 3D constructs of the same cell type. 


\section{Conclusions}

We have presented a platform for 2D cell culture with control and integrated measurement of oxygen availability. At present, it is capable of mimicking a continuous gradient through tumour environment using 2D culture. The platform maintains the advantages of standard 2D culture techniques and avoids complex processing that comes with 3D culture, while allowing addition of in-vivo features. The reusable system offers the ability to detach the PDMS chip for direct cell access after exposure to an oxygen gradient, providing the opportunity to image nanoscale cellular features with contact techniques such as AFM. This opens up possibilities for studying nanoscale features in different oxygen concentrations. While the system can be used for more accurate anti-cancer drug discovery and testing of combination therapies and hypoxia activated drugs, tissue engineering areas such as stem cell differentiation can profit from oxygen control as well. The only modification required to provide other physiologically relevant DO levels is to change the gas mixture. Furthermore, with additional inlet channels, the number of concentration regions can be increased towards a usable tumour-on-a-chip.

\section{Acknowledgements}

The authors thank Helen Devereux and Gary Turner for technical assistance as well as Kenny Chitcholtan and Elizabeth Dunn for providing cells. Financial support was provided by the Biomolecular Interaction Centre and the MacDiarmid Institute for Advanced Materials and Nanotechnology in form of a PhD scholarship.

\section{References}

[1] Yeom, C. J., Goto, Y., Zhu, Y., Hiraoka, M., Harada, H., Int. J. Mol. Sci. 13(11)

(2012) 13949-13965. Doi:10.3390/ijms131113949.

[2] Bristow, R. G., Hill, R. P., Nature Rev. Cancer 8(3) (2008) 180-192.

doi:10.103/nrc2344.

[3] Vaupel, P., Mayer, A., Cancer Metastasis Rev. 26(2) (2007) 225-239.

doi:10.1007/s10555-007-9055-1

[4] Brown, J. M., Wilson, W. R., Nature Reviews Cancer, 4(6) (2004) 437-447. doi:10.1038/nrc1367

[5] Sharkey, A. M., Day, K., McPherson, A., Malik, S., Licence, D., Smith, S. K., Charnock-Jones, D. S., The Journal of Clinical Endocrinology and Metabolism, 85(1) (2000) 402-409. doi:10.1210/jc.85.1.402

[6] Ware, M. J., Colbert, K., Keshishian, V., Ho, J. C.-S., Corr, S. J., Curley, S., Godin, B., Tissue Engineering. Part C, Methods, 4(August) (2016) 1-36.

doi:10.1089/ten.TEC.2015.0280 
[7] Gong, X., Lin, C., Cheng, J., Su, J., Zhao, H., Liu, T., Zhao, P., PLoS ONE, 10(6) (2015), 1-18. doi:10.1371/journal.pone.0130348

[8] Bauer, M., Su, G., Beebe, D. J., Friedl, A., Integrative Biology: Quantitative Biosciences from Nano to Macro, 2(7-8) (2010), 371-8. doi:10.1039/c0ib00001a

[9] Hirschhaeuser, F., Menne, H., Dittfeld, C., West, J., Mueller-Klieser, W., KunzSchughart, L. A., Journal of Biotechnology, 148(1) (2010), 3-15.

doi:10.1016/j.jbiotec.2010.01.012

[10] Khalili, A. A., \& Ahmad, M. R., International Journal of Molecular Sciences, 16(8) (2015), 18149-18184. doi:10.3390/ijms160818149

[11] Wang, L., Liu, W., Wang, Y., Wang, J., Tu, Q., Liu, R., Wang, J., Lab on a Chip, 13(4) (2013), 695-705. doi:10.1039/c2lc40661f

[12] Chen, Y.-A., King, A. D., Shih, H.-C., Peng, C.-C., Wu, C.-Y., Liao, W.-H., Tung, Y.C., Lab on a Chip, 11(21) (2011), 3626. doi:10.1039/c1lc20325h

[13] Lo, J. F., Sinkala, E., Eddington, D. T., Lab on a Chip, 10(18) (2010), 2394. doi:10.1039/c004660d

[14] Oppegard, S. C., Eddington, D. T., Biomedical Microdevices, 15(3) (2013), 407414. doi:10.1007/s10544-013-9737-0

[15] Nock, V., Murray, L., Samsuri, F., Alkaisi, M. M., Evans, J. J., Journal of Vacuum Science \& Technology B, Nanotechnology and Microelectronics: Materials, Processing, Measurement, and Phenomena, 28(6) (2010), C6K17-C6K22. doi:10.1116/1.3501342

[16] Nock, V., Blaikie, R. J., \& David, T., Lab on a Chip, 8(8) (2008), 1300-7. doi:10.1039/b801879k

[17] Nock, V., Blaikie, R. J., \& David, T., In 4th International Conference on Advanced Materials and Nanotechnology AIP Proceedings 1151 (2009), 67-70.

doi:10.1063/1.3203249

[18] Nishida, M., Hum Cell, 15(3) (2002) 104-117. doi:10.1111/j.1749-

0774.2002.tb00105.x

[19] L. H. Gevantman, https://sites.chem.colostate.edu/diverdi/all courses/CRC\%20reference\%20data/solubilit y\%20of\%20gases\%20in\%20water.pdf, accessed: 2017-10-08

[20] US Geological Survey, https://water.usgs.gov/software/DOTABLES/, accessed: 2017-10-08 
[21] Thermo Fisher Scientific, MEM, Hank's Balanced Salts, https://www.thermofisher.com/order/catalog/product/11575032, accessed: 2017-10-08

[22] Chitcholtan, K., Sykes, P. H., Evans, J. J., Journal of Translational Medicine, 10(1) (2012), 38-54. doi:10.1186/1479-5876-10-38

[23] Orcheston-Findlay, L., Hashemi, A., Garrill, A., Nock, V., PVP, Int. J. Nano. in press

[24] Inamdar, N. K., Griffith, L. G., Borenstein, J. T., Biomicrofluidics, 5(2) (2011), 022213-15. doi:10.1063/1.3576925

[25] Trédan, O., Galmarini, C. M., Patel, K., Tannock, I. F., Journal of the National Cancer Institute, 99(19) (2007), 1441-54. doi:10.1093/jnci/djm135

[26] Nock, V., Murray, L., Samsuri, F., Alkaisi, M. M., Evans, J. J., In Microelectronic Engineering 88(8) (2011), 1828-1831. doi:10.1016/j.mee.2010.12.042 